\title{
LA ENSEÑANZA INTEGRAL DE LA ARQUITECTURA, DESDE LA PERSPECTIVA DE LA SOSTENIBILIDAD AMBIENTAL
}

Artículo de Reflexión - Recibido 2 de Junio de 2015 - Aceptado 15 de Octubre 2015

\section{Bernardo Palacio Echenique}

Universidad del Atlántico. Barranquilla, Colombia. bpalacio@gmail.com

Para citar este artículo / to reference this article:

Palacio, B. (2016). La enseñanza integral de la Arquitectura, desde la perspectiva de la sostenibilidad ambiental. Módulo Arquitectura CUC, Vol.16 №1, 35-58.

\section{Resumen}

El presente artículo tiene como objetivo señalar la incidencia que en la enseñanza de la arquitectura tiene la sostenibilidad ambiental como elemento revaluador de los procesos académicos que se formalizan al interior del taller de proyecto, y que de una manera $u$ otra, pueden influir positivamente en el replanteamiento del modo como el docente conduce su asignatura proyectual, en la aspiración de fortalecer en sus estudiantes, criterios de diseño urbano arquitectónicos consecuentes con las políticas que en materia de sostenibilidad ambiental se han implementado en Colombia y a nivel internacional.

\section{Palabras Clave}

Enseñanza, Arquitectura, Taller, Proyecto, Sostenibilidad, Ambiental, Docente, Estudiante. 


\section{THE INTEGRAL TEACHING OF ARCHITECTURE, FROM THE PERSPECTIVE OF ENVIRONMENTAL SUSTAINABILITY}

\section{Abstract}

This article aims to point out the impact that the teaching of architecture has the environmental sustainability as a revaluador element of academic processes which are formalized project workshop inside, and that in one way or another, they can influence positively the rethinking of how the teacher leads her course project, in the aspiration to strengthen in students architectural urban design criteria consistent with policies that environmental sustainability have been implemented in Colombia and internationally.

\section{Keywords}

Education, Architecture, Workshop, Project, Sustainability, Environmental, Teaching, Student. 


\section{Introducción}

La enseñanza de la arquitectura aparece a lo largo de la historia como el lugar por excelencia en donde se confrontan las teorías constituidas, así como las historias de la arquitectura que narran su práctica, con una función social y con un papel a desempeñar en la conformación de la cultura, y ello conlleva la reflexión sobre el arquitecto a formar, a partir del precepto de que esa formación debe ser planeada en concordancia con el contexto inmediato en donde habrá de desempeñarse, y así evitar que las propuestas de planes de estudios sean obsoletas de origen.

En desarrollo de los deberes y derechos constitucionales (Senado de Colombia, 1991), la Ley 30 de 1992 (Congreso de Colombia, 1992) determina que es función del Estado propiciar el fomento de la calidad del servicio educativo; señala los conceptos de autonomía, en el sentido de que la institución de educación puede crear, organizar y desarrollar su programa académico. Se crea el Sistema Nacional de Acreditación con el objetivo fundamental de garantizar a la sociedad que las instituciones que hace parte de este Sistema cumplan con los más altos requisitos de calidad y que realicen sus propósitos y objetivos.
Además, dispone que la autoevaluación institucional es una tarea fundamental y permanente del proceso de acreditación y crea el Sistema Nacional de Información de la Educación Superior, SNIES, cuyo objeto es hacer planeación, monitoreo, evaluación, asesoría, inspección y vigilancia sobre las instituciones y programas de Educación Superior, para orientar a la comunidad sobre calidad, cantidad y características de esas instituciones y programas académicos (Ministerio de Educación Nacional, 2013).

\section{Principios y métodos de enseñanza de la arquitectura}

En Colombia se han llevado a cabo dos reformas estructurales del sistema educativo: La primera, cuando se organiza la educación bajo la responsabilidad del Ministerio de Instrucción Pública, con la Ley 39 de 1903 sobre la Instrucción Pública (Gutiérrez, Sánchez, \& Beltrán, 2009) en la que no se define qué es la educación ni mucho menos sus objetivos. La segunda reforma se realizó con la Ley 115 de 1994 (Congreso de Colombia, 1994) en la que se define la educación como un proceso de formación permanente, personal, cultural y social. 
De ello se concluye que en Colombia se trasladó la visión de la educación que se tenía hasta ese momento, como un proceso de instrucción a uno de formación del sujeto en un contexto sociocultural.

La tendencia que ha seguido la educación en el último siglo ha sido el otorgarle cada vez mayor protagonismo al estudiante en su proceso de formación: a comienzos del siglo XX la función del profesor era "decir" y la del alumno, "oír". Posteriormente el profesor toma en nombre de maestro y su función es "explicar", mientras que el alumno toma el nombre de estudiante y su función era "entender". A mediados de siglo, los llamados docentes deben "demostrar" a sus discentes mediante la "experimentación". Se pretende que el estudiante conozca el medio, se conozca a sí mismo, conozca los conocimientos y la manera adecuada para llegar a ellos. Implica todo un proceso de aprendizaje autónomo en el que él aprenda a aprender, siendo este un requisito para la formación por competencias.

Alrededor de 1975 con el surgimiento de las corrientes constructivistas la función de los educandos la construcción del conocimiento acompañados por el educador. A comienzos del siglo XXI surge un modelo en el que el educador cumple el papel de mediador en el proceso de formación que busca hacer del educando un líder agente de transformación competitivo en la sociedad (Gutiérrez, Sánchez y Beltrán, 2009).

\section{Panorama general de la enseñanza de la arquitectura}

Para todos los efectos legales, entiéndase por Arquitectura, la profesión a nivel universitario, cuya formación consiste en el arte de diseñar y crear espacios, de construir obras materiales para el uso y comodidad de los seres humanos, cuyo campo de acción se desarrolla fundamentalmente con un conjunto de principios técnicos y artísticos que regulan dicho arte (Consejo Profesional Nacional de Arquitectura y sus Profesiones Similares, 2012). Como tal, es un ejercicio cognoscitivo cuya manifestación es primeramente intelectual, no física, en cuanto está vinculada a la capacidad, exclusivamente humana, de aprender y comprender de forma consciente y libre, razón que la define como una praxis que se alimenta del saber (Mejía, 2008). 


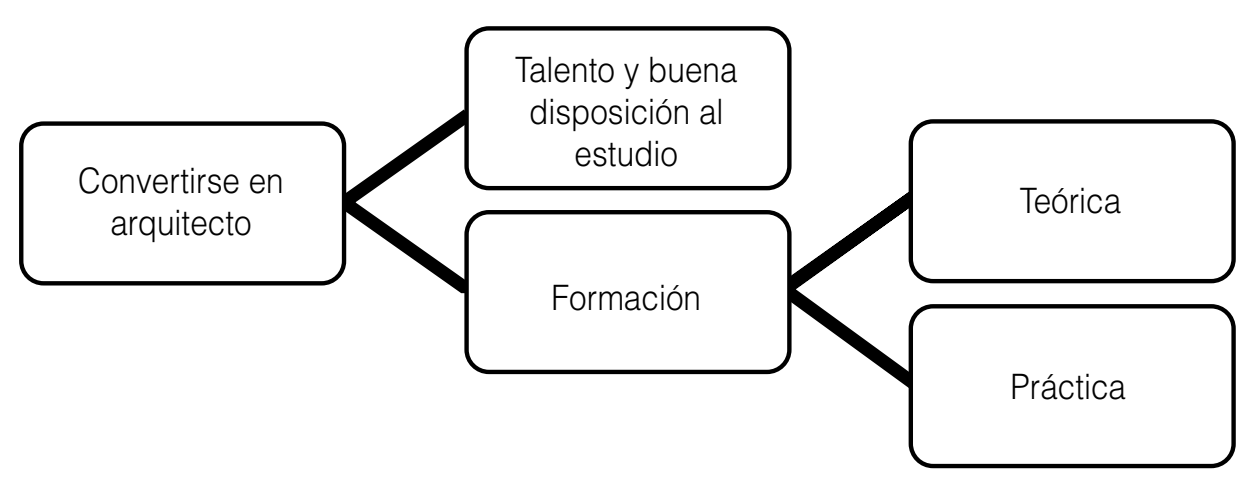

Gráfico 1. La formación del arquitecto

Fuente: http://etudesanciennes.revues.org/324

El arquitecto es un ser social y como tal aprende en su hogar, en su medio social y cultural y en el espacio reservado para él en el mundo universitario. Las escuelas o facultades de Arquitectura son socialmente responsables de la formación profesional de arquitectos. La educación entendida en toda su amplitud, es la responsable de formar personalidades. En el mundo de las cortes, de las aristocracias y de las altas burguesías, el arquitecto fue educado para atender las demandas de un espacio social y cultural exclusivo, distante de la condición del común de las gentes. En el mundo moderno, a partir de la Revolución Industrial, el profesional de la Arquitectura se educa para responder a un sinnúmero de demandas diferentes, unas de orden individual y privado, otras de orden social y público. La oferta universitaria debe nominalmente capacitar para afrontar esas demandas (Saldarriaga, 1996).

La pregunta de cómo se enseña arquitectura hoy es pertinente, ya que los límites de la actividad arquitectónica se expanden hacia campos que parecen nuevos y, al mismo tiempo, se cuestionan en aquellos que eran tradicionalmente propios (Gutiérrez et al, 2009). Por lo general, la estructuración de los planes de estudios de Arquitectura se plantea con base en formatos previamente establecidos, derivados por una parte, de la tradición reciente de la enseñanza de la Arquitectura y determinados, por otra, por exigencias de las instituciones universitarias (Saldarriaga, 1996). 
Pero ello, en parte, ha derivado en un distanciamiento entre el saber (sustentado en la teoría) y el hacer (sustentado en la práctica), con predominio evidente de este último, convirtiendo a la Arquitectura en un oficio más, restándole su carácter tradicional de espacio de pensamiento, menoscabando la creatividad, pues la fusión entre experiencia y conocimiento no se está realizando mediante un proceso simultáneo e integral. Ambas cosas se disgregan en compartimentos separados y divergentes. Existe un excesivo predominio de la enseñanza sobre el aprendizaje: el estudiante se forma en gran parte desde el temor y el protagonismo excesivo que sus profesores transmiten y ejercen, respectivamente (Meneses, 2013). El caso colombiano se ha inscrito en una tradición teórica y práctica heredada de la enseñanza académica europea que tuvo su centro en la Academia Francesa, y de las Escuelas desarrolladas posteriormente, que adoptaron el Movimiento Moderno en diferentes manifestaciones desarrolladas en Europa, los Estados Unidos y América Latina (García, 2002).

Las discusiones se han centrado en pequeñeces formales de currículos, sin entender que la búsqueda debe ser más profunda. Yala historia ha mostrado la relatividad de los conceptos que fundamentan la arquitectura depen- diendo de las condiciones temporo espaciales. Lo técnico, lo artístico y lo social varían, pero existe un factor común que ha definido la esencia de la arquitectura; éste es la habitabilidad como creación de espacio en el sentido fenomenológico, es el espacio que se crea en tanto el ser que lo habita y se identifica con él, ahora sí, desde lo tecnológico, lo artístico y lo funcional. En este sentido se debe educar para entender lo habitable y la habitabilidad, no para asumir prejuiciados contenidos que sólo permitan diseñar edificios (Castaño, Bernal, Cardona y Ramírez, 2005).

No obstante, la enseñanza de la arquitectura aparece como el lugar por excelencia en donde se confrontan las teorías constituidas, así como las historias de la arquitectura que narran su práctica, con una función social y con un papel a desempeñar en la constitución de la cultura. Y ello conlleva la reflexión sobre el arquitecto a formar, la cual es un tema central cuando se enfrenta la enseñanza de la arquitectura, que debe tomar partido desde el momento cuando se formula como tarea, por una concepción de la arquitectura, no solo en cuanto a disciplina, sino también en su función social (García, 2002). La razón de ser de los hechos arquitectónicos está dada por el pensamiento, por el contenido 
objetivo y claramente argumentativo de las teorías y los manifiestos que los originaron y que de alguna manera legitiman la práctica de la arquitectura (Mejía, 2008).

Seguramente en muchos de los programas existentes se ha intentado una y otra vez la elaboración de ejercicios académicos en los que se busca la elaboración de proyectos arquitectónicos constructivos. Seguramente también se habrán encontrado con las dificultades inherentes a dicho reto, derivadas de la complejidad del ámbito interdisciplinario. Esa dificultad ha superado en muchos casos los esfuerzos de las comunidades académicas, hasta el punto de aceptar, se podría decir, de común acuerdo, que la formación del arquitecto es suficiente cuando se encuentra capacitado para elaborar anteproyectos arquitectónicos (Ocampo, 2013).

En cuanto encargados de diseñar el espacio habitable, los arquitectos son en mayor o menor medida responsables de la calidad del entorno en que se desarrolla la vida. Preocupados por la degradación espacial que presentan la mayoría de nuestras ciudades, instituciones sociales, académicas y gremiales, están demandando una reorientación de la formación de los arquitectos con el fin de preparar profesionales que puedan mejorar la habitabilidad de los espacios públicos y privados de las urbes actuales. Indicadores de distinta naturaleza reflejan cotidianamente que los encargados de la configuración y gestión urbana han sido incapaces de dar soluciones adecuadas a las demandas que la sociedad les ha planteado y plantea. En reuniones académicas, debates, noticieros de televisión, editoriales de prensa y otros foros, es común que se enjuicie sin piedad la poca habitabilidad de nuestras ciudades. Razones no faltan: degradación estética y funcional del entorno, contaminación acústica y atmosférica, caos funcional, deficiencias de organización y construcción en las edificaciones e infraestructuras, falta endémica de viviendas dignas; en fin, una larga lista de problemas que configuran un panorama con el que es lógico que la mayoría de la población no se sienta a gusto (Goycoolea, 1998).

La enseñanza de la arquitectura está en crisis. Mantiene hoy en día la misma estructura básica de programa de hace cincuenta años. El taller como asignatura en la que convergen todas las demás, lugar de síntesis y creación, la columna vertebral de toda 
la carrera, desde siempre ha funcionado de la misma manera, a través del "desafío" o encargo del profesor. Este actúa como el "cliente" que señala el sitio, programa de necesidades y tiempo para el proyecto. Este esquema sólo cambia en magnitud y complejidad de año en año. El arquitecto debe poder gestionar su encargo. Diagnosticar el problema, detectar la necesidad, tener las herramientas que le permita organizar una gestión del proyecto, proponerlo y llevarlo a cabo. Un profesional que cree su trabajo, que esté antes del encargo y de otros profesionales con visión analítica y con capacidad de propuesta. Que se maneje en las leyes del mercado, que domine los indicadores económicos, versado en la técnica constructiva y en la ciencia de los números, para sólo entonces, generar el espacio que dé origen a la arquitectura (Quijano, 2001).

La sociedad latinoamericana $y$, especialmente la colombiana viven conflictos bastante fuertes por las desigualdades sociales, la corrupción, la violencia y el desplazamiento forzado, situaciones que ejercen una alta presión sobre la población, el entorno natural y las ciudades, pueblos $y$ veredas, que se transforman permanentemente y demandan cada vez rior y, principalmente en su periferia, habitan personas y familias en condiciones cada vez más precarias.

En este contexto, el proceso de formación del arquitecto debe apuntar en alto grado a la comprensión de estos fenómenos y a la búsqueda de alternativas de solución para el tema de los asentamientos humanos, espacio en el cual el arquitecto debe interactuar cada dia con mayor interés. Este es el reto de un profesional que, actuando con cultores de otras disciplinas, pueda convertirse en factor de cambio de ese medio social. En el lenguaje académico, esta perspectiva se denomina responsabilidad social universitaria (Londoño, 2008).

El arquitecto tiene una responsabilidad enorme: puede alegrar la vida de los seres que habitan el espacio arquitectónico o, por el contrario, hacerles la vida extremadamente aburrida o angustiosa. Otro de los elementos que en cierta medida no se puede medir en un espacio es el comportamiento de quien lo experimente. El espacio arquitectónico debe generar efectos que afecten los sentidos e influyan en las actitudes, debe ser un instrumento de intensificación de comportamientos y conducir ese comportamiento a un campo sensible (Cabas, 2011). 
El conocimiento en arquitectura exige un cambio en la mirada hacia la arquitectura y muy especialmente, en la mirada al Proyecto, tanto en su aproximación conceptual como en la acción proyectual, pues esta se sustenta sobre una actividad analítica, crítica y, por lo tanto, investigativa, que implica el compromiso con el conocimiento y su producción social y cultural, como posibles condiciones en las que se estructura el saber (Castellanos, 2012).

El Estado colombiano, mediante la Resolución 2770 de noviembre 13 de 2003, Art. 2, ha definido las características específicas de calidad para los programas de pregrado en Arquitectura que se oferten en el país. Se requiere que los programas de Arquitectura guarden coherencia con la fundamentación teórica, práctica y metodológica de la Arquitectura y con los principios y propósitos que orientan su formación desde una perspectiva integral, considerando, entre otros aspectos, las competencias y saberes que se espera posea el Arquitecto. Así mismo, deben guardar coherencia con la normatividad que rige su ejercicio profesional en el país (Ministerio de Educación Nacional, 2003).

\section{Concepto de proyectación}

La Real Academia de la Lengua Española reconoce el término proyección (Real Academia Española, 2001), pero el término proyectación pertenece al ámbito de la arquitectura, como un neologismo que proviene del verbo proyectar, que significa idear, imaginar, trazar, disponer de un plan y los medios para ejecutarlo. Tradicionalmente el término proyecto arquitectónico se considera como el conjunto de diseños, planos y cálculos, planta, alzadas, perspectivas, etc., que determinan todo lo necesario para la construcción de la obra arquitectónica (Jiménez, R. 2009). Proyectación puede entenderse como el acto de componer y con este, la forma o la construcción de una lógica de ordenar y relacionar los elementos y las partes de un edificio (Weiss, 2009).

La cuestión en torno al proyecto es cómo enseñar una técnica, un método, y cómo transmitir una cultura, un saber, en los talleres de arquitectura: la técnica por sí sola produce proyectos vacíos de contenido; la cultura por sí sola es conocimiento pero requiere de la acción, de la técnica, para convertirse en proyecto (Gamboa, 2009). 
Proyectar es crear un escenario virtual sobre el cual se sitúa hipotéticamente a los futuros ocupantes del espacio que se proyecta. Los planos, las maquetas y las animaciones por computador, además de apoyar la previsualización del edificio, sirven de marco de referencia para la localización de los destinatarios y sus diferentes perfiles, lo que supone el reconocimiento de algunas posibles actitudes y comportamientos que los habitantes puedan asumir una vez el edificio sea construido.

Es importante que el estudiante de Arquitectura comprenda que esta es una disciplina compleja, que el arquitecto no es hacedor de formas bonitas o de espacios agradables y bien compuestos -blancos o negros-, que el compromiso del arquitecto no es exclusivamente con un cliente ni con un usuario sino con seres humanos, con la sociedad, con la naturaleza. Que cada decisión que tome no ha de ser el producto de una fórmula sino de pensar la complejidad que constituye estructurar el "hábitat", en sustantivo (Herrera, 2011). Esa complejidad significa pasar por un intenso entrenamiento, dirigido a despertar y doblegar las condiciones innatas del estudiante de Arquitectura, para que desarrolle lo que el Arquitecto Urbanista Philippe Boudón llama "un pensar arquitectónico (citado por Moncada, 2012).

Gráfico 2. Proyectar: crear un escenario virtual

Fuente: http://piensartquilectura.blogspot.com/2011_07_01_archive.html
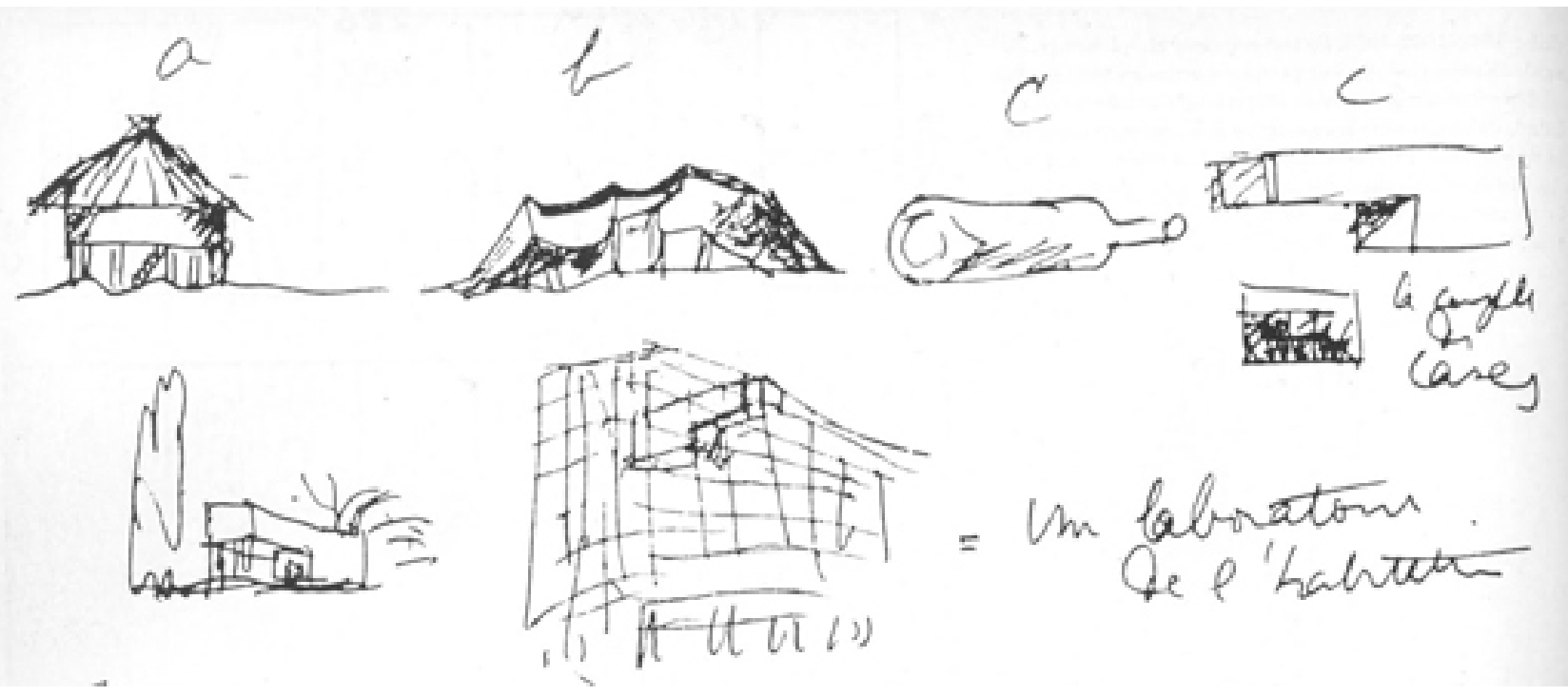

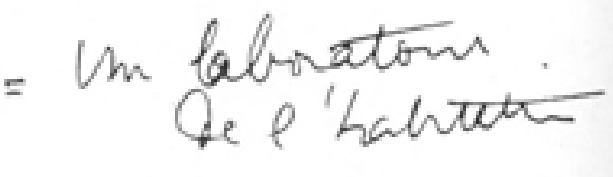




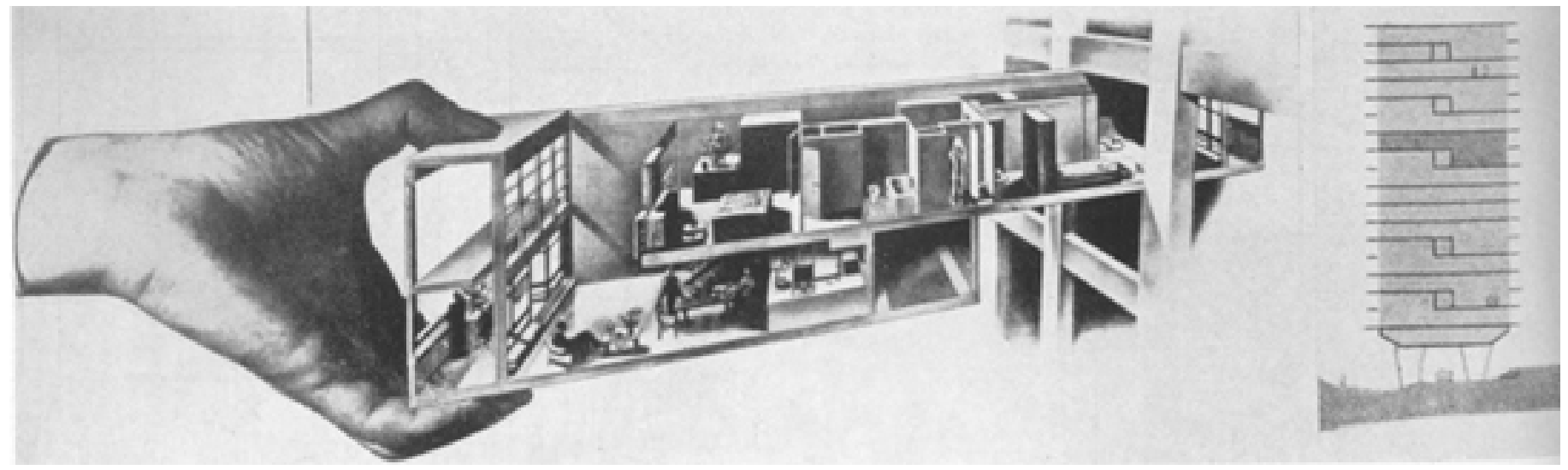

Gráfico 2. Proyectar: crear un escenario virtual

Fuente: http://piensartquilectura.blogspot.com/2011_07_01_archive.html

Proyectar es emplazar un edificio correctamente, satisfacer un programa y seleccionar el sistema constructivo más indicado, para que sus formas surjan de un proceso de proyectación a partir de lo esencial, lo que permite buscar referencias sin riego, teniendo la certeza de que el proyecto se enriquecerá, y conscientes de que los edificios siempre son "nuevos" pero las ciudades no, y por eso deben ser contextuales, y además, sostenibles (Barney, 2011). Proyectar es una actividad racional que sigue una lógica, y no el resultado circunstancial y caprichoso de una inspiración o de una idea intuitiva enmarcada dentro de una concepción artístico romántica de la creación; puesto que el proyecto es una construcción, es decir, un conjunto de partes que posee un orden en virtud de un propósito, es posible pensar que se pueda enseñar a hacer proyectos de manera racional en una escuela de arquitectura (Gamboa, 2009).

El proyecto como tal, surge en el momento en el que se establecen conceptos compositivos, funcionales, técnicos y referenciales. A partir de allí es fundamental la intuición, el aspecto creativo que vincula con el campo artístico y que da lugar a la formulación de soluciones o hipótesis, que luego se verifican en el proceso del proyecto. Es entonces la creatividad, como capacidad de combinación de elementos e interpretación de condiciones, la que permite que la arquitectura evolucione, se potencie y se renueve (Méndez, 2009). La teoría y la práctica son el contrapunto de la arquitectura. 
El proyecto y la obra. El anteproyecto es el sueño, el proyecto el despertar, y la construcción puede ser la pesadilla, pero también es la única realidad del edificio. Por esto los proyectos se nutren de ella al permitir llevar la experiencia de las construcciones realizadas a los nuevos proyectos (Barney, 2002).

El Taller de Diseño es un espacio integrador de saberes, disciplinas y ciencias, en el que se aborden temarios discursivos desde los cuales se puede fundamentar mejor la creación arquitectónica; es una forma para construir conocimiento y también sociedad y cultura (Meneses, 2013). Como fundamento se debe tener en cuenta el carácter interdisciplinario del trabajo proyectual, el cual no se traducen únicamente en la existencia de cursos separados para cada conocimiento necesario; es importante el desarrollo de una aproximación integradora en la que el proyecto sea el eje articulador de conocimientos diversos (Saldarriaga, 2009).

Por tal razón, se impone un aprendizaje que conduzca al estudiante desde un estado incipiente o elemental del conocimiento a uno de tipo más evolucionado, para que pueda urdir sus propios significados, desde la indagación y proposición de problemas reales (Meneses, 2013).
Como lo manifiesta el Arq. Carlos Niño Murcia (2002), el profesor de Taller debe contribuir a esa definición, a partir del conocimiento de la disciplina y del país, y sobre todo enseñar arquitectura, intuir sobre el trabajo de otros arquitectos, sus experiencias y el conocimiento de otras ciudades o latitudes, como también el manejo de las escaleras, de las luces o de las cubiertas, de la vegetación o los climas, de la cultura, la tradición o las vanguardias.

La enseñanza en los talleres de arquitectura parte de una premisa fundamental: se aprende a hacer arquitectura haciendo arquitectura; hacer proyectos es una actividad práctica que no se puede transmitir desde fuera, acudiendo únicamente a un manual de instrucciones, a un método que indique los pasos a seguir hasta llegar al final. Sin la práctica sería imposible aprender. Practicando de manera reiterada y con la guía de sus profesores, el estudiante va adquiriendo poco a poco unas destrezas que le permitirán luego desarrollar proyectos por su cuenta (Gamboa, 2009). La arquitectura es una profesión que se ocupa de labores que representan alto riesgo social, -pues así como puede proteger y dignificar la vida, puede someterla a condiciones de riesgo y a precariedades absolutas-, debe garantizarle a la comunidad que todos aquellos 
que ostentan la matrícula profesional, poseen solvencia e idoneidad ética y técnica para el ejercicio profesional (Pérez, 2002).

Tradicionalmente la metodología que se aplica en los cursos de taller está centrada por una parte, en la experiencia del profesor que repliega sus vivencias como estudiante de arquitectura y por otra, en que el profesor, que normalmente tiene una amplia experiencia como proyectista, repite en el curso su ejercicio profesional, con lo cual el taller de proyectos termina convirtiéndose en una réplica de su oficina particular (Villazón, Villate, \& Bravo, 2009). Cada programa asume el taller utilizando una modalidad $u$ otra, como lugar de síntesis disciplinar por excelencia, y adopta énfasis sobre los demás saberes que convergen en la formación (García, 2002).

El enseñar no es sino una actividad más del ser humano, inscrita en el permanente anhelo de la persona, de hacer llegar la experiencia vivida a los demás. Enseñar arquitectura no es sino intentar reproducir esa experiencia y los saberes adquiridos, de la manera más sencilla y clara posible. Enseñar a proyectar no es sino saber encontrar los recursos didácticos más efectivos para que la experiencia propia pueda ser experimentada por otros. Enseñar es dar seña del objeto a trabajar y los caminos para hacerlo (Weiss, 2009).

La pregunta que surge, en tanto el taller se plantea como el lugar disciplinar por excelencia, es ¿desde dónde se mira el taller? ¿Es éste un espacio de pensamiento y de transmisión de un oficio o es solamente un espacio de transmisión de un ofició? Desafortunadamente, aunque ha aumentado la conciencia sobre este punto, aún la gran mayoría de la enseñanza del taller se queda en este último nivel, dejando de lado el desarrollo del pensamiento que la producción de la arquitectura conlleva y que la sitúa como una de las artes, que a través de las configuraciones que logra, abre camino para nuevas posibilidades de ver el mundo (García, 2002).

\section{Concepto de sostenibilidad}

En la década del 70, la crisis generada por la disminución y encarecimiento en el abastecimiento del petróleo por parte de los países productores, puso de manifiesto la extrema dependencia a los combustibles fósiles como fuente energética, especialmente por parte de los países desarrollados. Esto generó un centramiento durante esa década en la búsqueda de soluciones a la escasez energética. 


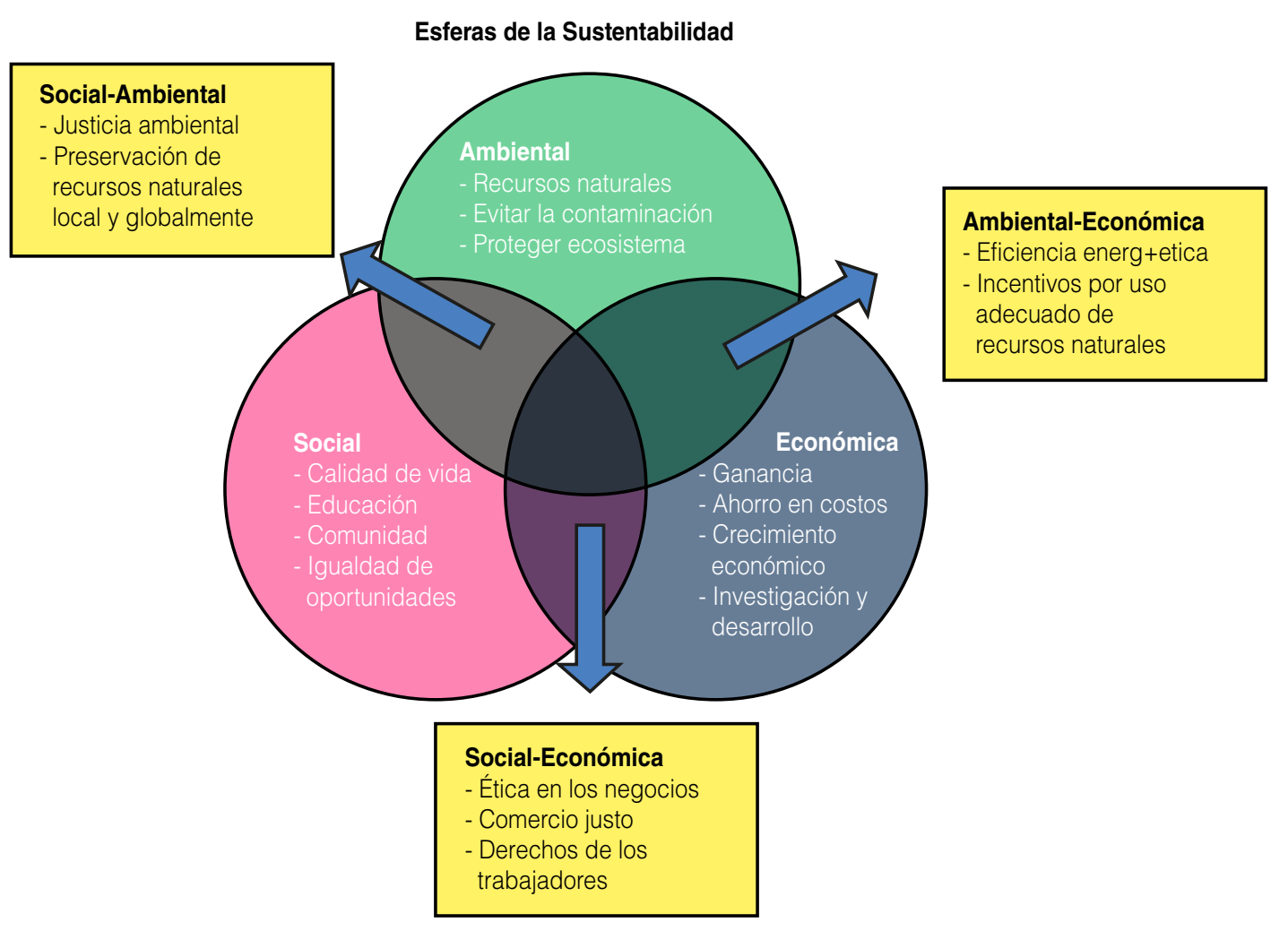

Gráfico 3. Desarrollo sostenible

Fuente: http://www.dforceblog.com/2012/12/21/sustentabilidad-vs-sostenibilidad/

En la disciplina de la arquitectura la búsqueda de alternativas a la escasez de recursos energéticos supuso una nueva mirada hacia las prácticas para la obtención del confort humano y del ahorro de energía, lo que dio como resultado la popularización del concepto de arquitectura bioclimática.
En los 80 se pone de manifiesto la evidencia del calentamiento global, con lo que se populariza el concepto de desarrollo sostenible. En los 90 fueron prioritarios los temas del agua y de su calidad; así como los relacionados con la protección del bosque tropical y de la biodiversidad. Hasta llegar al año 2000, en cuya primera 
década se abordaron temas como la salud de las ciudades, el diseño y la construcción sustentable, la sostenibilidad y la salud; así como la pobreza mundial y las enfermedades (Iglesias, 2012).

El concepto de Sostenibilidad hace referencia a la capacidad que haya desarrollado el sistema humano para satisfacer las necesidades de las generaciones actuales sin comprometer los recursos y oportunidades para el crecimiento y desarrollo de las generaciones futuras (Calvente, 2007).

\section{Arquitectura Sostenible}

Es la creación de espacios habitables, tanto a partir del diseño como de la edificación, en donde se aplican los criterios y premisas del desarrollo sustentable, por lo que en ésta los recursos naturales, económicos y humanos se manejan de forma tal, que se reducen el daño ambiental, reducción de contaminación del suelo, del agua (y su consumo) y del aire, mejoramiento del confort interno y externo del edificio (preferentemente de manera pasiva) y mejoramiento de la tecnología que da servicio en las edificaciones, como aparatos, máquinas y otros dispositivos tanto mecánicos como eléctricos. Lo anterior permite, en consecuencia, que se logre un mejoramiento del confort del edificio, $y$, asimismo, que se respete el entorno inmediato, es decir, el sitio o lugar de edificación (Hernández, 2008).

Para mejorar un modelo adecuado de relación sostenible hombrenaturaleza es necesario integrar además, aspectos socio-culturales, como las costumbres, tradiciones, tipología arquitectónica y organización social con la caracterización climática de la región donde se ubique el proyecto (Pérez, 2012). Entre más pronunciadas y adversas al hombre resulten las variables del entorno físico, más atenta y regionalmente caracterizada devendrá la arquitectura propuesta. La respuesta formal de la arquitectura al entorno, por su especial atención al trabajo de la envolvente, semeja la reacción de los seres humanos a las variaciones del entorno empleando diversas vestimentas (Aschner, 2009).

Cabe señalar que durante el proceso de diseño del proyecto se puede y debe prever, con suma atención, los problemas vinculados con el impacto ambiental que causará el edificio en todo su ciclo de vida. 


\section{Prácticas sostenibles en arquitectura}

Se estima que cerca de la mitad de todos los recursos no renovables consumidos a lo largo del planeta son utilizados en la construcción, haciéndola una de las industrias menos sostenibles en el mundo. La conciencia sobre la degradación del planeta, y del sector de la construcción como uno de los mayores consumidores a escala global de sus recursos, generó esfuerzos por conciliar la visión de desarrollo sostenible dentro de sus prácticas.

Esto motivó importantes cambios en el enfoque y la conceptualización sobre la arquitectura, la planificación y el diseño de las ciudades, que se han traducido en el surgimiento de lo que se conoce en la actualidad como la arquitectura, el urbanismo y la construcción sostenibles (Iglesias, 2012).

La fase de uso de las construcciones también posee un impacto ambiental específico, la energía consumida para iluminación y acondicionamiento ambiental es en gran medida controlada por decisiones de proyecto; como también la utilización de aguas servidas y contaminantes pavimentadas y compuestos orgánicos volátiles. La manutención y vida útil de las construcciones consume recursos en volúmenes aproximadamente iguales a los desprendidos en la fase de producción, que también generan polución (Sarria y López, 2012).

Como respuesta a esa situación, ha tomado fuerza la aplicación de prácticas sostenibles entendiéndolas como aquellas actividades complementarias que se realizan en cualquier proceso de producción, incluida la arquitectura, donde se toman en cuenta las premisas de sustentabilidad. En arquitectura, esas premisas son (Hernández, 2008): Sostenibilidad social, económica, cultural, de servicios y equipamiento, de espacios abiertos y urbanidad, geológica, hidrológica, paisajista, climática, alimenticia, sanitaria, energética y técnico-científica.

Partiendo de estas premisas, los arquitectos buscan contribuir al desarrollo sostenible al hacer ecológicos y sostenibles sus proyectos, y al utilizar, además, una herramienta fundamental para llevar a cabo dichas prácticas: el diseño sostenible en arquitectura. La arquitectura que ignora lo sustentable hiere al medio 
ambiente. Es una arquitectura que se apoya en bastones equivocados para funcionar-como por ejemplo, en las energías no renovables- y dilapida el futuro de las siguientes generaciones, perdiendo en su cinismo la oportunidad de ser verdaderamente bella, así esté decorada con maestría (Reinberg, 2009).

Tradicionalmente en el sector de la construcción se han utilizado materiales de carácter local tales como el ladrillo, la madera, etc., lo que se traducía en unos costes energéticos e impactos ambientales reducidos. Asimismo, existía una adaptación del diseño del edificio a las condiciones climáticas locales, lo que repercutía en una mayor calidad del edificio y un mayor confort térmico para los ocupantes. En la actualidad, el uso masivo de materiales de carácter global como el cemento, el aluminio, el PVC, etc., han causado un incremento notable en los costes energéticos y medioambientales (Zabalza, Díaz, Aranda y Scarpellini, 2014).

Lo sostenible poco se considera en los edificios que se diseñan en los talleres. Es preciso que consuman menos energía -pues hoy, irresponsablemente son los que más lo hacen- mediante su climatización pasiva y su iluminación natural, que ahorren agua -cada vez más escasa-, la reutilicen hasta donde sea posible y usen la lluvia; que no contaminen y procesen sus propias basuras; que sean higiénicos, seguros y accesibles a todos; que consideren su contexto, que sus espacios sean renovables sin producir tantos escombros y que sean reciclables y hasta degradables (Barney, 2011). Son tan costosos los desarrollos de los nuevos materiales y tan fundamental el reconocimiento de la limitación de los recursos, y es tan grande la responsabilidad hacia la problemática social global y tan esperanzadoras las perspectivas de mejoramiento del futuro a través de la actuación sostenible, que una arquitectura con conciencia no puede permanecer ignorante de la situación (Reinberg, 2009).

La humanidad asiste hoy a la desertización del planeta, a la arquitecturización del globo y a la construcción de la megalópolis. La gran ciudad se ha convertido en el escenario de los códigos; es la máquina que permite los contactos multiculturales dentro de la democracia; todo ello documentado con aridez acentuada en la obra de numerosos artistas. Pero el hombre trata de huir del sufrimiento y la desespera- 
ción de la existencia urbana creando burbujas antidepresivas. Así mismo, mediante la arquitectura, toma posesión del vacío y deja ver el horror que le tiene a la naturaleza en su estado primitivo. La ciudad, entonces, aparece como una obra contra la naturaleza, como la rectificación y la racionalización de lo natural -como si ello fuese incompleto o defectuoso (Chávez, 2008). La ciudad tiene la posibilidad de hacer un aporte de gran envergadura al desarrollo sostenible y a una manera correcta de formular la arquitectura y construirla. La arquitectura puede colaborar reduciendo el impacto ambiental y mejorando la calidad de los edificios, siguiendo tres reglas importantes: produciendo economías de energía, conservando los recursos, y concibiendo edificios sanos (Ramírez, 2010).

Es por ello que el arquitecto debe estudiar cuidadosamente el ambiente que será afectado en cada propuesta de tarea, evaluando los impactos ambientales en los ecosistemas involucrados, urbanizados o naturales, incluido el entorno socioeconómico, seleccionando la mejor alternativa para contribuir a un desarrollo ambientalmente sano y sostenible, con el objeto de lograr la mejor calidad de vida para la población, rechazando toda clase de recomendaciones en trabajos que impliquen daños evitables para el entorno humano y la naturaleza tanto en espacios abiertos, como en el interior de edificios, evaluando su impacto ambiental, tanto en corto como en largo plazo.

La enseñanza de la arquitectura desde la perspectiva de la sostenibilidad ambiental, puede ser más efectiva si se convierte en un suceso de contraste experimental, que comprenda el dominio de un discurso de tipo reflexivo-teórico; actuando como una síntesis generadora de conocimiento, tanto grupal como individual; permitiendo la integración de disciplinas diferentes, que puedan desembocar en el hecho arquitectónico de manera integral; logrando acumular y respetando, por memoria selectiva, el legado cultural que han construido las generaciones precedentes; intercambiando significados a través de procesos comunicativos, ya sean estos, gráficos, orales o escritos; optimizando procesos de construcción y apropiación del conocimiento; permitiendo la apertura de horizontes que hagan posible la afirmación del sujeto y su participación en la construcción de sociedad y cultura; y planteando propósitos educativos de indagación en problemas y situaciones reales, con impacto social (Meneses, 2013). 
Se requiere ahondar en la actualización, capacitación y formación de profesores que apoyen los programas curriculares en el proceso proyectual, para integrar al componente de proyectos el conocimiento medioambiental y sostenible a través del diseño arquitectónico y urbanístico que propenda por la optimización de los recursos y materiales; la disminución del consumo energético y el fortalecimiento en el uso de energías renovables, y la disminución de residuos y emisiones (Rodríguez, 2009). La docencia construye los procesos históricos de la academia. La realidad de la sociedad exige proyecciones sociales, antes que económicas, y posiciones de colectividad, antes que individuales o elitistas. Solo de esta manera el arquitecto ocupará el lugar que le corresponde ante el compromiso de una vanguardia creativa, consciente y consecuente (Pérez, 2010).

\section{Conclusión}

El compromiso ineludible de la academia es la formación de un arquitecto con responsabilidad social como estrategia para la transformación del hábitat en que intervenga, que asuma como propios los retos que impone la construcción de un hábitat sustentable, así como la construcción del conocimiento con pertinencia y acción social. Se trata de los compromisos de la academia por un desarrollo humano, integral y sostenible, respetuoso del Hábitat, o el sistema de circunstancias y elementos que, interrelacionados funcionan como una unidad, que proveen las condiciones y el medio necesario para el surgimiento y el desarrollo de la vida en el contexto arquitectónico (Decon, 2008).

En el campo de la arquitectura, como sucede en muchas otras áreas, la sostenibilidad está involucrada tanto en los aspectos más generales del quehacer arquitectónico (el proyecto y el diseño) como en los asuntos vinculados con costos e impacto ambiental y social. Las principales subdisciplinas en las cuales se apoya el arquitecto para crear se refieren a cuestiones técnicas de teoría, historia, diseño, tecnología, economía, gestión y, también, sustentabilidad (este último es tan importante como los demás); aspectos que, en conjunto, hacen integral la arquitectura (Hernández, 2008).

El diseño sostenible integra consideraciones de eficiencia en el uso de recursos y de la energía, ha de producir materiales sanos, ha de 
utilizar materiales ecológicos y debe considerar la sensibilidad estética que inspire, afirme y emocione (Ramírez, 2010), no solo teniendo en cuenta al hombre en sus dimensiones físicas, sino en su capacidad auditiva, resistencia a temperaturas, a la intensidad lumínica, en definitiva, en su capacidad para moverse dentro de un medio físico adecuado, que haga que su organismo esté convenientemente integrado y que no sufra deterioro.

En este contexto es fundamental el accionar del docente como facilitador, aprendiendo a aprovechar el impulso de los estudiantes por aprender, y la existencia del intercambio de las ganas de hacer. Más que un pensador crítico, el docente debe ser una persona habitualmente inquisitiva, bien informada, de mente abierta, flexible, justa cuando se trate de evaluar, honesta cuando confronte sus sesgos personales, prudente al emitir juicios, dispuesta a reconsiderar $y$, si es necesario, a retractarse; ordenada cuando se enfrente a situaciones complejas, diligente en la búsqueda de información relevante, razonable en la selección de criterios, persistente en la búsqueda de resultados tan precisos como las circunstancias, el
El docente debe estimular el pensamiento racional y no racional. Debe asimismo pensar que su objetivo final no es solamente el proyecto sino el pensamiento que lo sustenta y le da validez, con la pretensión de que algún dia sea arquitectura. Si es capaz de usar cualquier método que esté a su alcance para que la persona que lo acompaña emprenda su propia aventura y si es capaz de vivir de acuerdo con lo que dice pensar, entonces será llamado "maestro" (Herrera, 2011).

\section{Referencias}

Aschner, R. (2009). ¿Cómo concebir un proyecto arquitectónico? Bogotá, Revista de Arquitectura 05. Universidad de los Andes, Panamericana Formas e Impresos S.A. (30-41).

Barney, C. (2011). Arquitectura: arte y técnica, práctica y enseñanza. Bogotá, Revista Hito, Editorial Escala, 25 (45 - 52).

Cabas, M. (2011). Lo intangible del espacio arquitectónico. Bogotá, Revista Hito, Editorial Escala, 25 $(7-12)$.

Calvente, A. (2007). El concepto moderno de sustentabilidad. 
Universidad Abierta Interamericana. Recuperado el dia 12 de febrero de 2015 de http://www. sustentabilidad.uai.edu.ar/pdf/sde/ uais-sds-100002\%20\%20sustentabilidad.pdf

Castaño, J., Bernal, M., Cardona, D. y Ramírez, I. (2005). La enseñanza de la Arquitectura. Una mirada crítica. Manizales, Revista Latinoamericana de Estudios Educativos, Universidad de Caldas, 1 (125-147)

Castellanos, G. (2012). Construcción Lógica y Pensamiento Analógico en el Saber Pensar la Arquitectura. Revista Memorias. Seminario Internacional Arquitectonics Network en América. Universidad Santo Tomás, $(22$ - 23).

Chávez, J. (2008). La dimensión poética de lo natural. Bogotá, Revista Hito, Editorial Escala, 23 (115 - 120).

Consejo Profesional de Arquitectura y sus Profesiones Auxiliares (2012). Código de Ética para el ejercicio de la Arquitectura y sus Profesiones Auxiliares. Bogotá, Ediciones C.P.N.A.A.

Decon, C. (2008). El proyecto arquitectónico contemporáneo. Algunas consideraciones desde la pers- pectiva del hábitat. Bogotá, Revista Hito, Editorial Escala, 23 (15 - 18).

Gamboa, P. (2009). Por una enseñanza de la arquitectura más artesanal y menos artística. Bogotá, Revista DeArquitectura. Universidad de los Andes, Panamericana Formas e Impresos S.A. 05 (4 - 15).

García, B. (2002). Apuntes sobre la enseñanza de la Arquitectura. Bogotá, Revista Hito, Editorial Escala, 20 (9-22).

Goycoolea, R. (1998). La práctica y la teoría; o los desafíos de la enseñanza actual de la arquitectura. México, Universidad Veracruzana, Colección Pedagógica Universitaria, 29 (45- 72).

Gutiérrez, M., Sánchez, H. y Beltrán, F. (2009). Panorama sobre los modelos pedagógicos y curriculares de los programas de Arquitectura en Colombia y Latinoamérica. Bogotá, Editorial Libros del Páramo, Universidad La Gran Colombia.

Hernández, S. (2008). La sustentabilidad en la enseñanza de la arquitectura en México. México, Revista La Colmena, Universidad Autónoma del Estado de México, 59.

Herrera, V. (2011). Taxonomía, o los dolores del vacío para una sociedad de la información, para 
una sociedad del conocimiento. Bogotá, Revista Hito, Editorial Escala, 25 (28 - 37).

Iglesias, V. (2012). La tecnología como expresión de la relación de las sociedades con su entorno. Popayán, Revista Arcus, Institución Universitaria Colegio Mayor del Cauca, Utilgráficas Popayán, 2 (6 - 18)

Jiménez, R. (2009). El pensar y el aprendizaje de la arquitectura. Bogotá, Revista Hito, Editorial Escala, 24 (31 $-40)$.

Londoño, D. (2008). La formación en Arquitectura. Bogotá, Revista Hito, Editorial Escala, 23 (99 - 104).

Mejía, V. (2008). Pensando la Arquitectura. Bogotá, Revista Hito, Editorial Escala, 23 (93 - 98).

Méndez, R. (2009). Teoría y Práctica. Bogotá, Revista de Arquitectura. Universidad de los Andes, Panamericana Formas e Impresos S.A., 05 $(78-81)$.

Meneses, D. (2013), Breve Panorama Histórico de la Enseñanza y el Aprendizaje de la Arquitectura. Bogotá, Ediciones Universidad de América, Fundación Universidad de América.

Niño, C. (2002). Acerca de la enseñanza de la arquitectura ¿Quiénes somos? y ¿por qué...? Bogotá, Revista Hito, Editorial Escala, 20 (67 - 72).

Ocampo, J. (2013). Didáctica y percepción de la Arquitectura. Bogotá, Revista Hito, Editorial Escala, 27 $(10-11)$.

Pérez, J. (2002). Preparación para la educación y la práctica de arquitectura sin fronteras: riesgos $y$ oportunidades. Bogotá, Revista Hito, Editorial Escala, 20 (23 - 34).

Pérez, A. (2010). La participación como estrategia para una transformación sustentable del hábitat, dentro del "actuar" académico y profesional. Bogotá, Revista Traza. Universidad de La Salle, Editorial Kimpres Ltda., 01 (115 - 123).

Pérez, P. (2012). La arquitectura bioclimática como herramienta en la planificación de comunidades sostenibles en Oaxaca, México. Popayán, Revista Arcus, Institución Universitaria Colegio Mayor del Cauca, Utilgráficas Popayán, $2(27-39)$

Quijano, A. (2001). Sobre la enseñanza de la Arquitectura. Recuperado de el dia 8 de enero de 2015 de http://www.arqchile.cl/quijanoensenanza.htm

Ramírez, J. (2010). Código de Construcción Sostenible de Bogotá. 
Bogotá, Revista Arquitecto, Panamericana Formas e Impresión S.A., (51-54).

Real Academia Española (2001). Diccionario de la Real Lengua Española. España, Edición $N^{\circ} 22$. Recuperado el dia 18 de febrero de 2015 de http://lema.rae.es/ drae/?val=proyecci\%C3\%B3n

Reinberg, G. (2009). Apuntes sobre la arquitectura de la construcción ecológica. Bogotá, Revista Dearquitectura (04), Universidad de Los Andes, Panamericana Formas e Impresión S.A., (4 13).

República de Colombia. Congreso de la República, (1992). Ley 30 de diciembre 28 de 1992, Ley de la Educación Superior. Recuperado el dia 7 de noviembre de 2014 de http://www.mineducacion.gov.co/1621/articles86437_ Archivo_pdf.pdf

República de Colombia. Congreso de la República, (1994). Ley 115 de febrero 8 de 1994, Ley General de la Educación. Recuperado el dia 7 de noviembre de 2014 de http://www.mineducacion.gov. co/1621/articles85906_archivo_ pdf.pdf
República de Colombia. Ministerio de Educación Nacional. (2003). Resolución 2770 de noviembre 13 de 2003, Bogotá. Recuperado el dia 7 de noviembre de 2014 de http://www.cpnaa. gov.co/cpnaa/BancoMedios/Documentos\%20PDF/ resolucion\%20no.37\%20de\%20 2013.pdf

República de Colombia. Ministerio de Educación Nacional. (2013), Sistema Nacional de Información de la Educación Superior, SNIES. Recuperado el dia 8 de noviembre de 2014 de http://snies. mineducacion.gov.co/consultasnies/programa/buscar.jsp?con trol $=0.6045473162265452$

República de Colombia. Senado de la República. (1991). Constitución Política de Colombia. Recuperado el dia 7 de noviembre de 2014 de http://www.senado.gov. co/el-senado/normatividad/constitucion-politica

Rodríguez, A. (2009). Estrategias medioambientales como herramientas de diseño sostenible en la formación del arquitecto. Bogotá, Editorial Libros del Páramo, Universidad La Gran Colombia. 
Saldarriaga, A. (1996). Aprender Arquitectura, un manual de supervivencia. Bogotá, Editorial Impreandes

Sarria, J. y López, E. (2012). Desarrollo sustentable y Construcción sustentable. Popayán, Revista Arcus, Institución Universitaria Colegio Mayor del Cauca, Utilgráficas Popayán, 2 (19 - 26)

Villazón, R., Villate, C., y Bravo, G. (2009). El taller de proyectos de arquitectura: ¿ambiente de aprendizaje innovador? Bogotá, Revista DeArquitectura, Universidad de los Andes. Panamericana Formas e Impresos S.A., 05 (176 - 180).
Weiss, P. (2009). La enseñanza del proyecto arquitectónico. Bogotá, Revista DeArquitectura, Universidad de los Andes. Panamericana Formas e Impresos S.A., 05 (54 - 73).

Zabalza, I., Díaz, S., Aranda, A., y Scarpellini, S. (2014). Impacto de los materiales de construcción, análisis de ciclo de vida. España, Revista EcoHabitar, 13. Recuperado el dia 12 de febrero de 2015 de http://www.ecohabitar.org/ impacto-de-los-materiales-deconstruccion-analisis-de-ciclo-devida/ 